\title{
Respiratory morbidity and mortality of traumatic cervical spinal cord injury at a level I trauma center in India
}

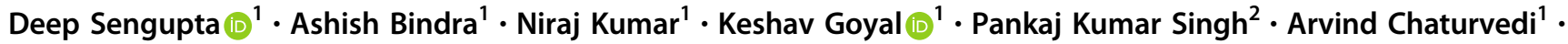 \\ Rajesh Malhotra ${ }^{3}$. Ashwani Kumar Mishra ${ }^{4}$
}

Received: 31 July 2020 / Revised: 9 November 2020 / Accepted: 26 November 2020

(c) The Author(s), under exclusive licence to International Spinal Cord Society 2021

\begin{abstract}
Study design Descriptive retrospective.

Objectives To evaluate the burden of respiratory morbidity in terms of ventilator dependence (VD) days and length of stay in neurotrauma ICU (NICU) and hospital, and to determine mortality in patients with traumatic cervical spinal cord injury (CSCI) in a low middle-income country (LMIC).

Setting Jai Prakash Narayan Apex Trauma Center (JPNATC), All India Institute of Medical Sciences (AIIMS), New Delhi, India.

Methods A total of 135 patients admitted with CSCI in the NICU between January 2017 to December 2018 were screened. Information regarding age, gender, American Spinal Injury Association (ASIA) impairment scale (AIS), level of injury, duration of VD, length of NICU, hospital stay, and outcome in terms of mortality or discharge from the hospital were obtained from the medical records.

Results A total of 106 CSCI patients were analyzed. The mean (SD) age of patients was $40( \pm 16)$ years and male: female ratio was 5:1. The duration of VD, duration of NICU, and hospital stay was a median of 8 days (IQR 1127), 6 days (IQR 1118), and 15 days (IQR 3127) respectively. Mortality was 19\% (20/106). The mortality was significantly associated with poorer AIS score, VD, and duration of ICU and hospital stay. All patients were discharged to home only after they became ventilator-free.

Conclusions The ventilator burden, hospital stay, and mortality are high in patients with CSCI in LMICs. Poor AIS scores, prolonged VD, ICU and hospital stay are associated with mortality. There is a need for comprehensive CSCI rehabilitation programs in LMICs to improve outcome.
\end{abstract}

$\triangle$ Ashish Bindra

dr_ashi2208@yahoo.com

1 Neuroanaesthesiology and Critical Care, Jai Prakash Narayan Apex Trauma Center, All India Institute of Medical Sciences, New Delhi, India

2 Neurosurgey, Jai Prakash Narayan Apex Trauma Center, All India Institute of Medical Sciences, New Delhi, India

3 Jai Prakash Narayan Apex Trauma Center, All India Institute of Medical Sciences, New Delhi, India

4 Biostatistics, National Drug Dependence Treatment Center, All India Institute of Medical Sciences, New Delhi, India

\section{Introduction}

Traumatic cervical spinal cord injury (CSCI) has extensive respiratory morbidity which may culminate in prolonged ventilator dependence (VD), extensive utilization of resources, and high mortality [1-3]. Literature from highincome countries (HICs) shows better outcomes and recovery in specialized spinal cord injury rehabilitation centers [4]. Comprehensive multidisciplinary management of CSCI is fraught with challenges in low middle-income countries (LMICs) like India with little literature quantifying the respiratory burden, length of stay, and mortality in this cohort of patients [5]. In absence of specialized spinal cord injury (SCI) rehabilitation centers, these patients stay for long times in trauma centers, which not only presses the acute care system but also delays the overall rehabilitation and may affect mortality. 
Our study highlights the burden imposed by CSCI in terms of ventilator dependence and length of neurotrauma intensive care unit (NICU) and hospital stay, and determines survival in this cohort of patients, at a level I trauma center in an LMIC without a specialized spinal cord injury rehabilitation facility or program. We reviewed the records of patients with exclusive CSCI admitted to our NICU. Data regarding age, sex, American Spinal Injury Association (ASIA) impairment scale (AIS), level of injury, duration of VD, length of NICU stay, hospital stay, and in-hospital mortality was obtained.

\section{Methods}

The study was conducted at Jai Prakash Narayan Apex Trauma Center (JPNATC), All India Institute of Medical Sciences (AIIMS), New Delhi, India after approval from the Institute Ethics Committee (IEC-101/01.02.2019, RP - 20/ 2019). AIIMS is an Indian institute of excellence in medical education, patient care, and medical research. JPNATC is a Level I trauma center attached to AIIMS. It's a public center providing care to trauma victims from across the country, especially North India, at a very subsidized cost. The Center has a total bed strength of 240. It's a high volume center with more than 75,000 patient visits each year. The center caters to all types of trauma patients. It has a dedicated Neurotrauma intensive care unit (NICU), managed by neuroanaesthesiologists and neurosurgeons. NICU mainly deals with the management of traumatic brain and spinal cord injury. Neuroanaesthesiologists/intensivists are available round the clock and are responsible for critical care and ventilatory management of patients in the NICU. NICU has an adequate nurse-patient ratio, and automated vital signs and data capture monitoring. Other specialties like physiotherapy and infection control are also available. Due to high flow of neurotrauma patients and a limited number of beds in NICU, ventilated patients are also managed in wards with round-the-clock nursing and provision of on-call neuroanaesthesiologists for ventilator and hemodynamic management and physiotherapists for chest physiotherapy. However, our center has no spinal cord injury rehabilitation program or a long-term rehabilitation facility attached to it.

This retrospective analysis included all patients with exclusive CSCI admitted in NICU from January 2017 to December 2018. Pediatric patients ( $<17$ years), and those with a head injury, polytrauma, patients not admitted to NICU, patients with incomplete medical records, and patients not discharged during the study period were excluded. All patients were managed according to the hospital treatment protocol and advanced trauma life support (ATLS) guidelines [6]. AIS was used to assess the severity of CSCI and was determined from the radiological investigations (anteroposterior and lateral radiographs, computerized tomography or magnetic resonance imaging) and clinical examination at admission. Injury involving $\mathrm{C} 1-\mathrm{C} 4$ level was considered as upper cervical spine injury and C5-C8 level was considered as lower cervical spine injury. Information regarding age, gender, duration of ventilator dependence, duration of NICU, and hospital stay was obtained from medical records. The outcome was noted in terms of mortality or discharge from the hospital. Patients received cervical traction in the $\mathrm{ED}$ or after admission in NICU as per the neurosurgical team consideration. Patients were informed about the high risks associated with the surgery as well as the requirement of prolonged mechanical ventilation even post-surgery. They were also informed about the conservative management option; the final decision was made in consultation with both surgical team and patient's choice. Those patients who were very sick in terms of cardiorespiratory status, and not fit to undergo anesthesia and surgery, were managed conservatively. Weaning approaches used were either pressure support ventilation (PSV) or progressive ventilator-free breathing (PVFB) on T-piece [7, 8]. Weaning was commenced when the patient met the following criteria: Vital capacity: $>10 \mathrm{~mL} / \mathrm{Kg}$ (IBW), Respiratory rate: 10-12 breaths/min, Minute ventilation: $<10 \mathrm{~L} / \mathrm{min}, \mathrm{PaO} 2>80 \mathrm{mmHg}$ with $\mathrm{FIO} 2$ at $\leq 0.4$, $\mathrm{PaCO} 2<45 \mathrm{mmHg}$, rapid shallow breathing index (RSBI): $<105$, clear or improving chest radiographs, stable vital signs and no complications like fever, sepsis, etc. PVFB weaning consisted of a ventilator-free time that was gradually increased which allowed a period of spontaneous breathing (training) followed by time on the ventilator. The training units (5-10 min per hour and minimum 8, max 12) were progressively increased each day until ventilator free breathing (VFB) was gradually achieved. VFB was considered to be achieved when the patient comfortably tolerated $24 \mathrm{~h}$ without the requirement of mechanical ventilation. Patients were followed to hospital discharge or in-hospital death, whichever was earlier.

Descriptive statistics were used to present epidemiological data. IBM Statistical Package for Social Sciences (SPSS) version 21 for Windows ${ }^{\circledR}$ was used for data analysis. Continuous normally distributed variables (age) are expressed as a mean and standard deviation; skewed data (VD, NICU, Hospital stay) are expressed as a median and interquartile range (IQR). Categorical variables are expressed as number percentage [ $n(\%)]$. Student's $t$-test was used to compare continuous data and Kruskal-Wallis equalityof-populations rank test was used for skewed data. Categorical variables were compared using Chi-square or Fisher's exact test. A $p$ value of $<0.05$ was considered statistically significant. The risk of mortality was estimated by performing the binary logistic regression analysis. The outcome variable considered in the analysis was in-hospital 
mortality (0-survived, 1-died). The independent predictors were -gender (0:Female, 1:Male), AIS score ( 0 : AIS-A, 1:AIS-B), level of injury (0: Lower Spine, 1: Upper Spine), age ( $Z$-score), ventilator dependence ( $Z$-score), duration of ICU stay ( $Z$-score), and duration of hospital stay ( $Z$-score); where 0 and 1 denote the reference and the risk group. The results of the binary logistic regression were interpreted in terms of the estimated odds ratio (OR) and the $95 \%$ Confidence Interval (95\% CI), under both the univariate and multivariable model (adjusted for age and gender). Under the multivariable model, Bonferroni's correction in the $p$ value was made for multiple comparisons. The survival analysis was done between motor complete (AIS-A/B) and motor incomplete (AIS-C/D) and neurologically intact (AIS-E) injuries estimating the likelihood of requirements for ventilation (measured in days). The event of interest was survival, and the outcome of interest was in-hospital mortality. The likelihood of a period of ventilation was assessed through the Log Rank Test.

\section{Results}

In this retrospective analysis, medical records of a total of 135 patients admitted with CSCI to NICU from January 2017 to December 2018 were screened. Out of these, 29 patients were excluded (22 pediatric and 7 adults with incomplete records). A total of 106 patients were included in the final analysis. Patient characteristics and injury severity values are shown in Table 1 . The total duration of VD was a median of 8 days (IQR 1127). The duration of VD was highest in AIS-A patients [median 21 days (IQR 3127)], followed by AIS-B [median 19 days (IQR 2,60)], AIS-C [median 9 days(IQR 2,31)], AIS-D [median 3 days (IQR 116)] and AIS- E [median 2 days $(2,5)$ ]; $(p<0.001)$ (Table 1) (Fig. 1).

Number of patients with injury involving upper (C1-C4) and lower (C5-C7) cervical spine was 55 (51.9\%) and 51 (48.1\%), respectively. Mean age, VD, ICU, and hospital stay, and outcome is described in Table 2. There was no difference in ventilator dependence, duration of ICU stay,
Table 1 Patient parameters according to AIS grade.
Fig. 1 Ventilator dependence in patients with Cervical Spinal cord injury. Graph showing duration of ventilator dependence in days in patients with cervical spinal cord injury (CSCI). $\mathrm{X}$ axis displays the number of ventilator dependent days. Y axis displays severity of injury. *Total number of patients studied (All CSCIs), American Spinal Injury Association Scale A (AIS A), American Spinal Injury Association Scale B (AIS B), American Spinal Injury Association Scale C (AIS C) and American Spinal Injury Association Scale D (AIS D).

\begin{tabular}{llllllll}
\hline Parameters & All patients & AIS $^{\mathrm{b}}$-A & AIS $^{\mathrm{b}}$-B & AIS $^{\mathrm{b}}$-C & AIS $^{\mathrm{b}}$-D & AIS $^{\mathrm{b}}$-E & $p$ value \\
\hline Total number of patients (N $\left.\mathrm{v}^{\mathrm{a}}\right)$ & 106 & $37(35 \%)$ & $8(8 \%)$ & $12(11 \%)$ & $32(30 \%)$ & $17(16 \%)$ \\
Male: Female & $5: 1$ & $30: 7$ & $7: 1$ & $10: 2$ & $29: 3$ & $13: 4$ & \\
Age(years): Mean (SD) & $40(16)$ & $36(14)$ & $48(15)$ & $34(13)$ & $41(18)$ & $47(13)$ & 0.02 \\
Ventilator dependence (days) Median (IQR) & $8(1127)$ & $21(3127)$ & $19.5(2,60)$ & $9.5(2,31)$ & $3(116)$ & $2(2,5)$ & $<0.001$ \\
ICU stay(days) Median (IQR) & $6(1118)$ & $9(2,118)$ & $14.5(8,22)$ & $6(3,18)$ & $3.5(114)$ & $2(1,5)$ & $<0.001$ \\
Hospital Stay(days) Median (IQR) & $15(3127)$ & $23(3127)$ & $19(17,60)$ & $18(10,37)$ & $7(3,26)$ & $6(4,11)$ & $<0.001$ \\
\hline
\end{tabular}

${ }^{\mathrm{a}} N$ Number of patients in each AIS grade.

${ }^{\mathrm{b}}$ AIS American Spinal Injury Association Impairment Scale.

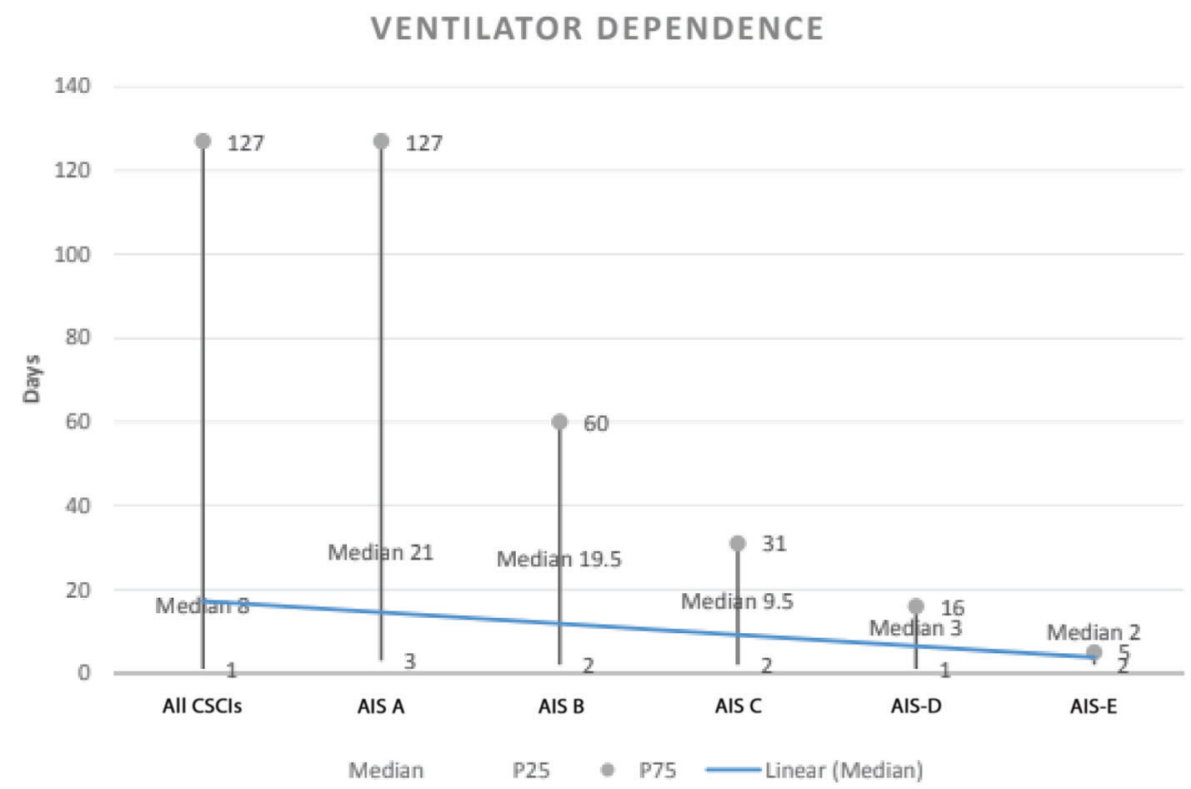


duration of hospital stay and mortality between the upper and lower cervical spine injury group.

The total duration of ICU stay was a median of 6 days (IQR 1118). The median duration of ICU stay in AIS-A, B, $\mathrm{C}, \mathrm{D}$, and $\mathrm{E}$ is shown in Table 1. The overall duration of hospital stay was a median of 15 days (IQR 3127). The median duration of hospital stay in different AIS impairment scales is shown in Fig. 2.

Duration of stay in ICU in $\mathrm{C} 1-\mathrm{C} 4$ level patients was median 5 days (IQR 1,45) and in C5-C8 level was median 7 days (IQR 2,118); $(p-0.206)$. The duration of hospital stay in C1-C4 level patients was median 11 days (IQR 3$65)$ and in C5-C8 level patients was median 19 days (IQR 3127); ( $p-0.136)$ (Table 2).

A total of 94 (89\%) patients out of 106 underwent a surgical correction in our study (AIS-A 32\%, AIS-B 7.4\%, AIS-C $11.7 \%$, AIS-D $31 \%$ and AIS-E $18 \%$ of total patients

Table 2 Patient parameters according to the level of injury.

\begin{tabular}{lclc}
\hline Parameters & $\begin{array}{l}\text { Upper cervical } \\
\text { spine (C1-C4) }\end{array}$ & $\begin{array}{l}\text { Lower cervical } \\
\text { spine (C5-C7) }\end{array}$ & $p$ value \\
\hline $\begin{array}{l}\text { Total number of } \\
\text { patients }(N)\end{array}$ & $55 / 106(51.9 \%)$ & $51 / 106(48.1 \%)$ & 0.582 \\
$\begin{array}{l}\text { Age (years) } \\
\text { Ventilator dependence(days) }\end{array}$ & $39.38 \pm 17.05$ & $41.15 \pm 14.29$ & 0.379 \\
$\begin{array}{l}\text { Mean } \pm \text { SD } \\
\text { Median (Inter }\end{array}$ & $13.2 \pm 16.21$ & $18.64 \pm 25.01$ & 0.154 \\
Quartile Range) & $6(1-60)$ & $11(2-127)$ & \\
ICU stay (days) & $8.47 \pm 9.30$ & $10.39 \pm 16.72$ & 0.206 \\
& $5(1-45)$ & $7(2-118)$ & \\
Hospital & $17.45 \pm 16.02$ & $22.9 \pm 24.0$ & 0.136 \\
stay (days) & $11(3-65)$ & $19(3-127)$ & \\
Death & $10 / 55(18.18 \%)$ & $10 / 51(19.6 \%)$ & 0.859 \\
Survived & $45 / 55(81.82 \%)$ & $41 / 51(80.4 \%)$ & 0.849 \\
\hline
\end{tabular}

who underwent surgery). Forty-three patients (40.5\%) underwent tracheostomy (AIS-A 27, B-7, C-6, D-3, and E-0 patients, respectively).

The results of the binary logistic regression analysis revealed that mortality was significantly associated with AIS-A, ventilator dependence ( $z$-score), ICU stay ( $z$-score), and hospital stay ( $z$-score). The univariate model [Odds Ratio (95\% CI), p], for age [0.822(0.532, 1.462), 0.627], gender $[0.486(0.149,1.686), 0.232]$ and level of injury [0.911 $(0.344,2.411), 0.851]$ was found to be statistically insignificant. Multivariable model (adjusted for age and gender) was also developed to find out the odds of the mortality. It was found that individuals in the category of AIS-A had 45 times more odds for mortality. Moreover, with increasing duration of ventilator dependence, NICU stay, and hospital stay, the estimated odds for mortality was three (3.298), eight (7.668), and two times (2.411). The independent variables remained statistically significant even after adjustment in the $p$ value for the multiple comparison (Table 3).

Mortality during hospital stay was $19 \%$ (20/106). The Mean (SD) age of patients who died was $39( \pm 16)$ years and patients who survived were $41( \pm 16)$ years. AIS-A patients had the highest mortality with $43 \%$ deaths followed by AISB patients (37\%) (Table 4). Cause of death was cardiorespiratory collapse secondary to severe cervical spinal cord injury (5/20), acute respiratory distress syndrome. and ventilator-associated pneumonia leading to sepsis $(5 / 20)$, septicemia (9/20; implant infection 1/9, catheter-associated bloodstream infection 5/9 and catheter-associated urinary tract infection 3/9) and hepatic failure (1/20). The median duration of VD was higher in patients who died as compared to the patients who survived [(median 21 days (IQR 3127 ) vs 5 days (IQR 1, 59); $(p<0.001)]$. The duration of ICU stay and hospital stay was also higher in patients who
Fig. 2 Hospital stay in patients with cervical spinal cord Injury. Graph showing duration of hospital stay in patients with cervical spinal cord injury (CSCI). X Axis displays duration of hospital stay in days. Y Axis displays severity of injury. *Total number of patients studied (All CSCIs), American Spinal Injury Association Scale A (AIS A), American Spinal Injury Association Scale B (AIS B), American Spinal Injury Association Scale C (AIS C) and American Spinal Injury Association Scale D (AIS D).

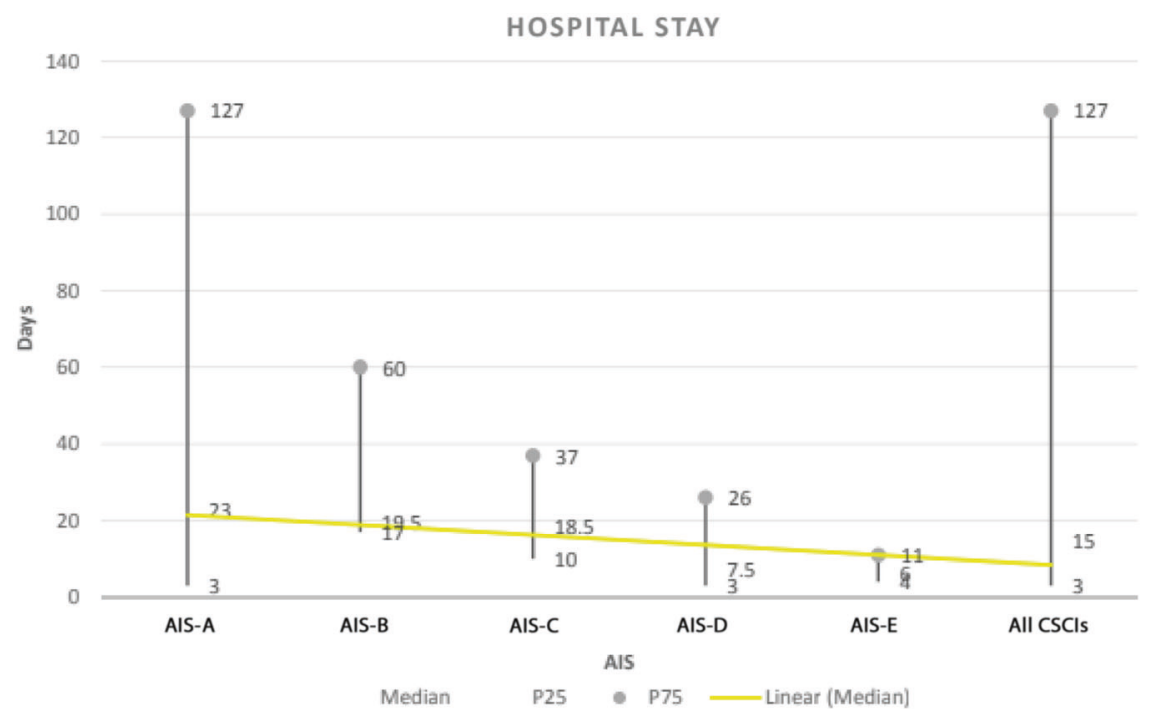


Table 3 Binary logistic regression analysis for dependent variable mortality.

\begin{tabular}{|c|c|c|c|c|c|c|}
\hline \multirow[t]{2}{*}{ Independent variable } & \multicolumn{3}{|c|}{ Univariate model } & \multicolumn{3}{|c|}{ Multivariable model ${ }^{\mathrm{a}}$} \\
\hline & Odds ratio & $95 \%$ CI & $P$ value & Odds ratio & $95 \% \mathrm{CI}$ & $P$ value $^{\mathrm{b}}$ \\
\hline AIS-A ${ }^{c}$ & 43.846 & $5.572,345.003$ & 0.003 & 45.043 & $5.621,360.949$ & $<0.001$ \\
\hline Ventilator dependence & 3.078 & $1.680,5.636$ & $<0.001$ & 3.298 & $1.734,6.271$ & $<0.001$ \\
\hline Z-Score & & & & & & \\
\hline ICU stayZ-score & 5.956 & $2.251,15.755$ & $<0.001$ & 7.668 & $2.699,21.783$ & $<0.001$ \\
\hline Hospital stay Z-Score & 2.322 & $1.384,3.895$ & $<0.001$ & 2.411 & $1.406,4.136$ & $<0.001$ \\
\hline
\end{tabular}

${ }^{a}$ Adjusted for Gender and $\mathrm{Age}_{\mathrm{Z}-\mathrm{Score}}$.

${ }^{\mathrm{b}}$ Significant after adjustment in $P$ value for multiple comparison.

${ }^{\mathrm{c}}$ AIS-B reference category.
Table 4 Comparison of outcome across parameters.

\begin{tabular}{lll}
\hline Parameters & Death & Survived \\
\hline Total number of patients(percentage) & $20 / 106(19)$ & $86 / 106(81)$ \\
Age (years) & $39 \pm 16$ & $41 \pm 16$ \\
Ventilator dependence (days) & $22(3127)$ & $6(1,59)$ \\
ICU stay (days) & $22(3127)$ & $4(1,24)$ \\
Hospital stay (days) & $22(3127)$ & $13(3,65)$ \\
AIS-A & $16 / 37(43 \%)$ & $21 / 37(57 \%)$ \\
AIS-B & $3 / 8(38 \%)$ & $5 / 8(63 \%)$ \\
AIS-C & $0 / 12$ & $12 / 12(100 \%)$ \\
AIS-D & $1 / 32(3 \%)$ & $31 / 32(97 \%)$ \\
AIS-E & $0 / 17$ & $17 / 17(100 \%)$ \\
\hline
\end{tabular}

died as compared to the patients who survived [median 21 days (IQR 3127) vs 4 days (IQR 1,24); $(p<0.001)$ and (median 21 days (IQR 3127) vs 12 days (IQR 3,65); ( $p$ $-0.038)$ respectively]. Survival improved from AIS A to E $(p<0.001)$ (Table 2).

The KM survival probability estimated difference in the survival probability across AIS lesions $(p-0.21)$ and the numbers at risk are shown in Fig. 3. Patients were not followed up after discharge from the hospital.

\section{Discussion}

CSCI was associated with long VD, prolonged stay in hospital, and mortality in our study. Limited data quantifies the same in CSCI patients undergoing treatment in LMICs. In our study, a total of 135 patients with exclusive CSCI were admitted to NICU over 1 year. Most of the patients were male (89/106) in the age group 25-55 (40 \pm 16$)$ years which is similar to reported literature [9, 10]. The total duration of mechanical ventilation was median 8 (IQR 1127) days. AIS correlates directly with the development of respiratory complications $[11,12]$. In our study, AIS-A patients had the longest duration of VD, up to 127 days, which is long as compared to reported literature for other trauma patients admitted at our hospital. The duration of ventilator dependence was similar between upper and lower cervical spine injury. A study reported a median (IQR) duration of mechanical ventilation and ICU stay of $5(2,10)$ and 5(2,9) days respectively, in 4692 trauma patients admitted to JPNATC over 2 years [13]. Gupta et al. compared the management of severe traumatic brain injury at JPNATC with Harborview Medical Center (HMC) in the USA [14]. ICU and length of hospital stay (LOS) at JPNATC and HMC was mean (SD) 6.1(2.1) vs 18.2(21.1) and 13.5 (9.9) vs 28.2 (24.3) days respectively. In our present study, the length of hospital stay was a median of 15 days (IQR 3127). In our study, due to the limited number of beds in the NICU, following initial optimization and stabilization some of the patients were managed in the high dependency units instead of NICU so that the bed could be made available for a more critical neurotrauma patient.

Wang et al reported a VD of $10.9 \pm 6.9$ days in acute CSCI patients [15]. In their study, $<50 \%$ of patients required mechanical ventilation at the time of admission and they ascribed higher extubation rate to a Glasgow Coma Scale of 15 and administration of comprehensive multidisciplinary care. In a retrospective analysis of SCI patients admitted to a state-designated Level 1 trauma center in a HIC, Mathew et al. reported a hospital stay of 14.1 days and ventilator dependence of 3.5 days, with acute in-hospital mortality of $<10 \%$ [16]. Most of the data regarding stay are reported from rehabilitation centers. An Italian multicenter prospective observational study showed a median stay of 135 days $(4,387)$ in rehabilitation centers for patients with CSCI [17]. The duration of stay correlates with the severity and level of injury [18]. However, in our study, duration of ICU and hospital stay was higher in severe AIS but was similar in upper and lower cervical spine injury. Hospital stays of up to 250 days have been reported in the literature $[16,19,20]$. Such prolonged stays at acute care facilities have been cut short in HICs by shifting the patient to rehabilitation centers.

Specialized spinal cord injury rehabilitation centers in HICs are comprised of multiple disciplines including 
Fig. 3 Kaplan Meier Survival Curve. Plot of Kaplan-Meier product limit estimates of American Spinal Injury Association Scale. A and B patients versus American Spinal Injury Association Scale C,D. Survival probability estimated across severity of injury had a $p$ value of 0.21 .

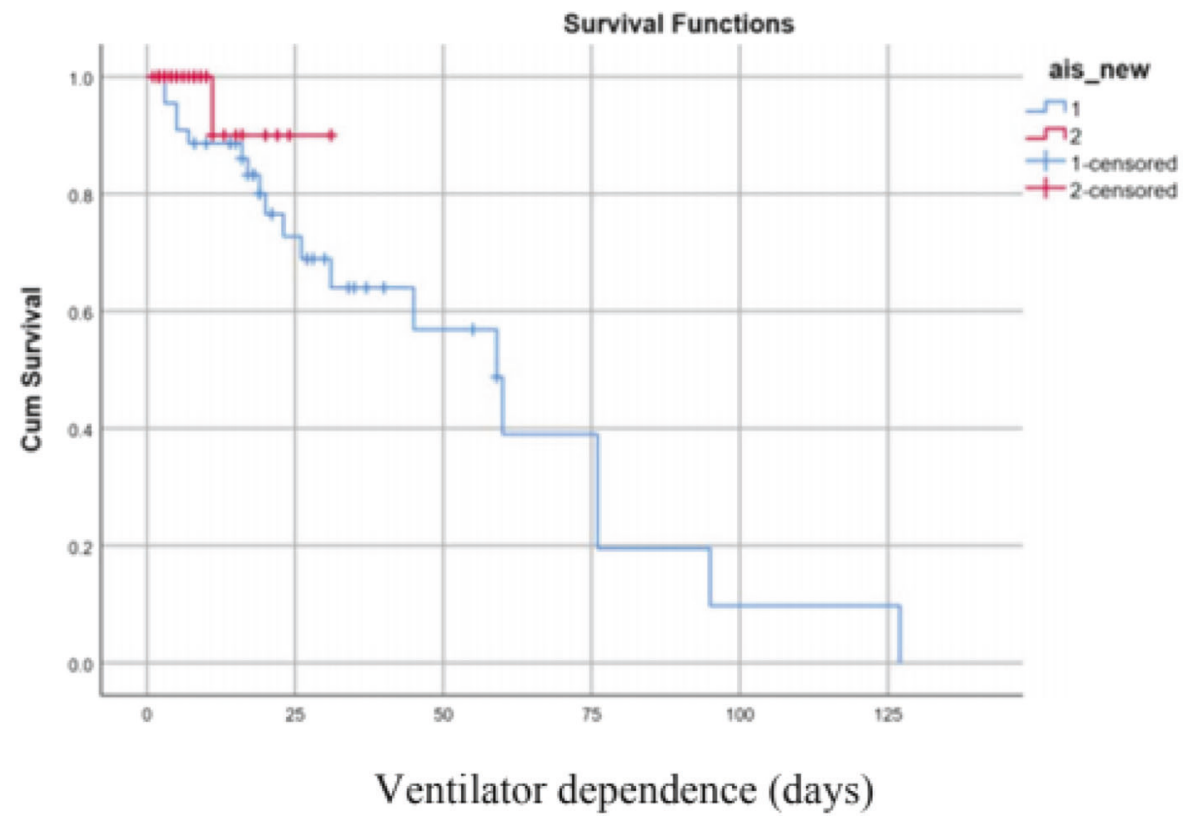

rehabilitation nurses, nurse technicians, physical therapists, occupational therapists, therapeutic recreation therapists, clinical psychologists, respiratory therapists and functional mobility training specialists (transfer training, joint mobilization, stretching, core stabilization exercises, standing, and/or gait training) besides clinical care managers like surgeons and intensivists and access to imaging facilities [4]. Easy referrals from acute care settings to long term rehabilitation is an integral part of care in patients with cervical spine injury. Besides these facilities, advanced multi-model rehabilitation, transcranial magnetic stimulation, and epidural electrical stimulation combined with dynamic task-specific rehabilitation training and intelligent therapies like stem cells, is being explored for better functional outcomes [21]. Patients with acute cervical spine injury spend a substantial time in ICU due to cardiovascular instability and other acute issues before they get transferred to a rehabilitation unit. Ideally rehabilitation should start in NICU once these patients become medically stable. An individualized plan should be developed depending upon need of each patient. Physicians, nurses, respiratory therapists, physical and occupational therapists, speech pathologists, dieticians, and rehabilitation psychologists are vital parts of the rehabilitation team in NICU. Similar facilities of care for traumatic spinal cord injury are missing in LMICs. LMICs lag behind HICs in domains of home care facilities and comprehensive functional, social, and economic rehabilitation [5]. In LMICs, patients with CSCI stay in acute care hospitals with limited rehabilitation facilities as long as they are sick and ventilator dependent, and get discharged home once they are stable and ventilator-free. These lacunae in care in LMICs may lead to higher morbidity and poorer quality of life [22].
All-cause mortality in our study was 19\% (20/106) with the highest number of deaths occurring in AIS-A patients (43\%), followed by AIS-B (37\%) patients. However, 98\% of AIS- C, D \& E patients survived and were discharged from the hospital. The level and severity of the injury, the requirement of vasopressors, and the long duration of mechanical ventilation are the primary causes of morbidity and mortality in the acute phase of SCI [8, 23-26]. We found AIS-A score, long VD and long ICU and hospital stay were associated with mortality. In our study, the survival analysis didn't reveal statistically significant results. However, it suggests that patients with motor complete injuries experienced higher mortality as compared to patients with motor incomplete injuries. The comparative appraisal of two curves (Fig. 3) clearly reveals that the majority of patients who died within a short span of ventilation had a motor complete injury (AIS-A/B), whereas only one patient died in the other group (AIS-C/D/E). These findings suggest that further studies with a larger sample size are required for the association of AIS lesions with the outcome. Patients with ventilator-dependent SCI are 39.5 times more likely to die during the first year after the injury [27]. Life expectancy for individuals with SCI in LMICs is shorter than for individuals with SCI in HICs [22]. A review found that patients with SCI and their families in an LMIC setup face a plethora of challenges including a small number of specialized SCI centers, limited access to rehabilitation services, and inadequate management of complications [22].

As reported in the literature, HICs, integrated in hospital rehabilitation centers, help in the institution of specialized respiratory rehabilitation programs and in comprehensive 
multidisciplinary care to aid in overall recovery [28]. In our study, all patients were discharged home once they were ventilator-free. Such patients stay at home with inadequate home care facilities. The long-term challenge is the management of ventilator, tracheostomy, pressure care, and autonomic functions, which is the reason that spinal cord units evolved. Since family is the main care provider at home, getting them involved from the start gives them an understanding and adaption of what will be required in the long term. Transfer of patients to an integrated multidisciplinary specialized center for care has shown to decrease overall mortality, length of stay in acute care facilities, and overall complications [4]. P Rinkaewkan et al. in their retrospective study found that the effectiveness of rehabilitation for SCI inpatients was directly associated with the capacity to institute an intensive rehabilitation program [29]. In the absence of similar facilities, patients in LMICs like India remain at the acute care setting for the entire duration of management. The prolonged stay of patients in acute care settings results in a scarcity of ICU and hospital beds. Shifting to integrated rehabilitation facilities will not only improve outcomes but will also offload the burden on acute care traumatology units. Though we believe that management of acute spinal trauma at Level 1 and 2 trauma centers is better than non-trauma centers, upgrading the rehabilitation facilities in LMICs will further lead to decreased mortality and morbidity. Challenges are increasing with an alarming rise in the incidence of SCI, huge unmet requirements of rehabilitation, increasing costs, and deficiency in trained health care personals [30]. Perhaps a program of community-based rehabilitation could be established, with the voluntary help of allied health workers from established spinal injuries units around the world.

In this study we quantified the effect of CSCI only on respiratory morbidity in terms of $\mathrm{VD}$, length of bed occupancy, and in-hospital mortality in one of India's level-1 trauma centers. Our study is a single-center retrospective analysis. Our mortality data are only relevant to trauma patients who got admitted to NICU, JPNATC, AIIMS, and cannot be reflective of all. We didn't study comorbid medical conditions, such as smoking, chronic obstructive pulmonary disease, pneumonia, and heart disease, which may have influenced the VD and mortality. Patients were followed up to discharge only.

\section{Conclusion}

Severe CSCI was associated with prolonged duration of VD, hospital stay, and mortality. High AIS, prolonged VD, NICU and hospital stay are associated with high mortality. In our study, after stabilization and liberation from ventilator, patients were discharged home. We highlight the need for comprehensive respiratory rehabilitation and posthospital care programs in LMICs. Further, research initiatives and feasibility studies should be undertaken for improving comprehensive care facilities in LMICs and to study the mid and long-term outcomes in these patients.

\section{Data availability}

The datasets generated and/or analyzed during the current study are available from the corresponding author on reasonable request.

Author contributions DS: Designing, screening the records, Data collection, and report writing. AB: Designing, Data collection, analyzing the data and writing. KG: Designing and writing. NK: Designing and writing. PKS: Contributed to designing of the retrospective analysis, analyzing the results and making the report. AC: Contributed to designing of the retrospective analysis, analyzing the results and making the report. RM: Contributed to designing of the retrospective analysis, analyzing the results and making the report. AM: Contributed to data interpretation and Statistical analysis.

\section{Compliance with ethical standards}

Conflict of interest The authors declare no competing interests.

Ethical approval The study was approved by the Institute Ethics Committee, All India Institute of Medical Sciences, New Delhi, India via letter no IEC-101/01.02.2019, RP - 20/2019.

Publisher's note Springer Nature remains neutral with regard to jurisdictional claims in published maps and institutional affiliations.

\section{References}

1. Mock C, Joshipura M, Arreola-Risa C, Quansah R. An estimate of the number of lives that could be saved through improvements in trauma care globally. World J Surg. 2012;36:959-63.

2. Singh R, Dhankar SS, Rohilla R. Quality of life of people with spinal cord injury in Northern India. Int $\mathrm{J}$ Rehabil Res. 2008;31:247-51.

3. Spiegel DA, Gosselin RA, Coughlin RR, Joshipura M, Browner $\mathrm{BD}$, Dormans JP. The burden of musculoskeletal injury in low and middle-income countries: Challenges and opportunities. J Bone Jt Surg - Ser A. 2008;90:915-23.

4. Parent S, Barchi S, LeBreton M, Casha S, Fehlings MG. The impact of specialized centers of care for spinal cord injury on length of stay, complications, and mortality: a systematic review of the literature. J Neurotrauma. 2011;28:1363-70. https://doi.org/ 10.1089/neu.2009.1151

5. Chhabra HS, Sharma S, Arora M. Challenges in comprehensive management of spinal cord injury in India and in the Asian Spinal Cord network region: findings of a survey of experts, patients and consumers. Spinal Cord. 2018;10:71-7.

6. Galvagno SM, Nahmias JT, Young DA. Advanced trauma life support ${ }^{\circledR}$ Update 2019: management and applications for adults and special populations. Anesthesiol Clin. 2019;37:13-32.

7. Füssenich W, Hirschfeld Araujo S, Kowald B, Hosman A, Auerswald M, Thietje R. Discontinuous ventilator weaning of patients with acute SCI. Spinal Cord. 2018;56:461-8. 
8. Galeiras Vázquez R, Rascado Sedes P, Mourelo Fariña M, Montoto Marqués A, Ferreiro Velasco ME. Respiratory management in the patient with spinal cord injury. BioMed Res Int. 2013. https://doi.org/10.1155/2013/168757

9. Lalwani S, Singh V, Trikha V, Sharma V, Kumar S, Bagla R, et al. Mortality profile of patients with traumatic spinal injuries at a level I trauma care center in India. Indian J Med Res. 2014;140:40-45.

10. Aleem IS, DeMarco D, Drew B, Sancheti P, Shetty V, Dhillon M, et al. The burden of spine fractures in India: a prospective multicenter study. Glob Spine J. 2017;7:325-33.

11. Loss SH, De Oliveira RP, Maccari JG, Savi A, Boniatti MM, Hetzel MP, et al. The reality of patients requiring prolonged mechanical ventilation: a multicenter study. Rev Bras Ter Intensiv. 2015;27:26-35.

12. Berlowitz DJ, Wadsworth B, Ross J. Respiratory problems and management in people with spinal cord injury. Breathe. 2016;12:328-40.

13. Soni KD, Kaushik G, Gupta A, Singh V, Kumar S, Sagar S. From emergency department to intensive care unit, does the delay matter to trauma patients? J Emerg Trauma Shock. 2018;11:189-92. https://doi.org/10.4103/JETS.JETS_132_17

14. Gupta D, Sharma D, Kannan N, Prapruettham S, Mock C, Wang $\mathrm{J}$, et al. Guideline adherence and outcomes in severe adult traumatic brain injury for the CHIRAG (collaborative head injury and guidelines) study. World Neurosurg. 2016;89:169-79. https://doi. org/10.1016/j.wneu.2015.12.097

15. Wang HC, Chen KY, Lin YT, et al. Factors associated with prolonged mechanical ventilation and reventilation in acute cervical spinal cord injury patients. Spine. 2020;45:E515-E524. https://doi.org/10.1097/brs.0000000000003302

16. Bank M, Gibbs K, Sison C, Kutub N, Papatheodorou A, Lee S, et al. Age and other risk factors influencing long-term mortality in patients with traumatic cervical spine fracture. Geriatr Orthop Surg Rehab. 2018;9:2151459318770882 https://doi.org/10.1177/ 2151459318770882

17. Franceschini M, Bonavita J, Cecconi L, et al. Traumatic spinal cord injury in Italy 20 years later: current epidemiological trend and early predictors of rehabilitation outcome. Spinal Cord. 2020;58:768-777. https://doi.org/10.1038/s41393-020-0421-y

18. Pickett GE, Campos-Benitez M, Keller JL, Duggal N. Epidemiology of traumatic spinal cord injury in Canada. Spine (Philos
Pa 1976). 2006;31:799-805. https://doi.org/10.1097/01.brs. 0000207258.80129 .03

19. Liebscher T, Niedeggen A, Estel B, Seidl RO. Airway complications in traumatic lower cervical spinal cord injury: a retrospective study. J Spinal Cord Med. 2015;38:607-14.

20. Conradsson D, Phillips J, Nizeyimana E, Hilliar C, Joseph C. Risk indicators of length of acute hospital stay after traumatic spinal cord injury in South Africa: a prospective, population-based study. Spinal Cord. 2019;57:763-9. https://doi.org/10.1038/s41393-019-0286-0

21. Zheng Y, Mao YR, Yuan TF, Xu DS, Cheng LM. Multimodal treatment for spinal cord injury: a sword of neuroregeneration upon neuromodulation. Neural Regen Res. 2020;15:1437-50. https://doi.org/10.4103/1673-5374.274332

22. Øderud T. Surviving spinal cord injury in low income countries. Afr J Disabil. 2014;3:80 https://doi.org/10.4102/ajod.v3i2.80

23. Neumann CR, Brasil AV, Albers F. Risk factors for mortality in traumatic cervical spinal cord injury: Brazilian data. Crit Care. 2009;67:67-70.

24. Higashi T, Eguchi H, Wakayama Y, Sumi M, Saito T. Risk factors associated with mortality after traumatic cervical spinal cord injury. OTA Int. 2018;1:e003.

25. Chamberlain JD, Meier S, Mader L, Von Groote PM, Brinkhof MWG. Mortality and longevity after a spinal cord injury: Systematic review and meta-analysis. Neuroepidemiology 2015;44:182-98.

26. Shao J, Zhu W, Chen X, et al. Factors associated with early mortality after cervical spinal cord injury. J Spinal Cord Med. 2011;34:555-62. https://doi.org/10.1179/2045772311Y.0000000024

27. Shavelle RM, DeVivo MJ, Strauss DJ, Paculdo DR, Lammertse DP, Day SM. Long-term survival of persons ventilator dependent after spinal cord injury. J Spinal Cord Med. 2006;29:511-9. https://doi.org/10.1080/10790268.2006.11753901.

28. Zakrasek EC, Nielson JL, Kosarchuk JJ, Crew JD, Ferguson AR, McKenna SL. Pulmonary outcomes following specialized respiratory management for acute cervical spinal cord injury: a retrospective analysis. Spinal Cord. 2017;55:559-65. https://doi. org/10.1038/sc.2017.10

29. Rinkaewkan P, Kuptniratsaikul V. The effectiveness of inpatients rehabilitation for spinal cord patients in Siriraj hospital. Spinal Cord. 2015;53:591-7. https://doi.org/10.1038/sc.2015.8

30. Renaud R, Locke H, Hariharan R, Chamberlain M, O'Connor R. Developing a spinal cord injury rehabilitation service in Madagascar. J Rehabilitation Med. 2018;50:402-5. 